\title{
LA FORMACIÓN DE INVESTIGADORES EN EDUCACIÓN: RETOS Y PERSPECTIVAS PARA AMÉRICA LATINA EN EL SIGLO XXI
}

\author{
Carlos Rincón Ramírez \\ Universidad Autónoma de Chiapas, México
}

\section{PRESENTACIÓN}

Los problemas de rezago educativo en América Latina, caracterizado por altos índices de analfabetismo, deserción y abandono escolar, insuficiente cobertura, bajos porcentajes de eficiencia terminal, bajo promedio de escolaridad, se encuentran asociados a las condiciones de pobreza, miseria, desnutrición y explotación que vive la mayor parte de la sociedad en esta región del continente americano. El modelo educativo imperante en estos países está asociado al modelo de desarrollo económico y al tipo de hombre y mujer que se desea formar; por tanto, al tipo ideal de sociedad que se debe construir. Las condiciones educativas de nuestras sociedades, son justamente el reflejo de las condiciones de deterioro social en que se encuentran y de la implantación de un modelo de economía que no responde a las necesidades de los sectores sociales más desprotegidos.

Lamentablemente en la mayor parte de Latinoamérica, se ha impuesto un proyecto económico que ha privilegiado el interés mercantil y financiero de los grandes consorcios tanto nacionales como internacionales. Así, la educación como proyecto de desarrollo y progreso social, se ha subordinado a proyectos que tienden a fortalecer las economías trasnacionales del modelo neoliberal de crecimiento.

Los gobiernos locales han concebido a la educación como un problema presupuestal que implica un gasto no redituable para las economías nacionales. Se ha priorizado la inversión en enclaves y desarrollos productivos, sin atender los graves y lacerantes problemas de hambre y pobreza que impera en la mayor parte de las sociedades de estas naciones. Las políticas sociales han servido de paliativo ${ }^{1}$ y en poco han contribuido a generar procesos de desarrollo humano más justos, equitativos, igualitarios y democráticos que garanticen la formación integral de las nuevas generaciones y condiciones de equilibrio de beneficio social entre los que más tienen y los más desprotegidos.

Desafortunadamente, las políticas educativas que sustentan los gobiernos en América Latina, hacen casi imposible que los modelos educativos actuales, atiendan los complejos problemas de la educación. Una visión de intervención diferente, que conciba a la educación como una inversión para generar las transformaciones necesarias que garanticen un desarrollo humano con alto sentido social, ${ }^{2}$ significa un replanteamiento de las estrategias de atención a dicha problemática. Replanteamiento que parte precisamente de las políticas oficiales educativas para construir una propuesta alternativa que posibilite la solución de la problemática educativa.

\footnotetext{
${ }^{1}$ En el caso particular de México, el Programa de Educación, Salud y Alimentación (PROGRESA) que otorga apoyos económicos a estudiantes de educación primaria y secundaria que habitan en comunidades de alta marginación y el Programa de solidaridad (PROGRESA) que también otorga apoyos económicos a familias de escasos recursos, son dos ejemplo de cómo los gobiernos aplican políticas de antiprobeza que finalmente, son falacias ya que las áreas marginadas cada día se incrementan y los indicadores estratégicos de superación de la pobreza: tasas de decrecimiento en el número de pobres, disminución de la intensidad media de la pobreza, incremento del poder adquisitivo de las familias, decrecimiento de los índices de marginación, por citar algunos, no son atendidos adecuadamente. Incluso, la política social aplicada es individualista ya que beneficia a las personas en detrimento de los grupos sociales.
} 
Atender los diversos problemas de la educación, que laceran a la mayoría de las naciones de Latinoamérica, con los modelos educativos vigentes, es continuar manteniendo las condiciones de rezago. Se requiere diseñar nuevas estrategias educativas que reconceptualicen la formación de profesionales de calidad. Y ante la dimensión de los diversos problemas que están subyaciendo a la educación en esta parte del mundo, una alternativa es formar investigadores educativos. La formación de investigadores en educación debe diseñarse como una propuesta alternativa de profesionales que, además de atender con profesionalismo los problemas educativos, adquieran un compromiso social y humano que les permita participar en la formación de los más altos valores universales.

\section{LOS HECHOS EDUCATIVOS CONTEMPORÁNEOS ANTE UN NUEVO MILENIO}

En las postrimerías del siglo $\mathrm{XX}$ y en los albores del $\mathrm{XXI}$, se manifestaron y continúan expresándose, una diversidad de sucesos y acontecimientos sociales, culturales, políticos, científicos, étnicos, ideológicos, religiosos que están modificando los patrones socioculturales que tradicionalmente se habían instaurado en las sociedades modernas. Estos procesos fueron convulsionados por la desaparición de la Guerra Fría y el eclipsamiento de la bipolaridad entre los dos grandes bloques económicos que habían dominado al mundo después de la Segunda Guerra Mundial. La desaparición del llamado poder socialista de la esfera internacional, benefició el fortalecimiento de la hegemonía capitalista norteamericana y, a su vez, provocó el debilitamiento de los países del llamado segundo mundo. Además, trastocó el estado contemporáneo de las relaciones que se encontraban vigentes entre las naciones en las distintas regiones mundiales.

En este panorama diversos bloques económicos se conformaron y consolidaron: la Unión Europea en Europa, y en América Latina la Comunidad Andina de Naciones, Mercado Común de América del Sur, Comunidad del Caribe, Mercado Común Centroamericano, así como el Tratado de Libre Comercio entre Estados Unidos de Norteamérica, Canadá y México, por mencionar algunos de ellos. La presencia de nuevos actores globales: corporaciones trasnacionales, agencias multilaterales y mecanismos supranacionales se han orientado al fortalecimiento del modelo económico neoliberal y, como consecuencia, ha influido en el debilitamiento de los Estados-nación (Souza Silva, 1999) como protagonistas centrales de los cambios contemporáneos.

Estos procesos de mundialización de las economías, así como implicaron que las relaciones de interdependencia entre los países ricos y pobres se ahondaran cada vez más y que las posibilidades de crecimiento de los pueblos en vías de desarrollo, se alejaran en la medida en que el control económico se encuentra en pocas manos, tuvieron repercusiones en las formas tradicionales de producir conocimiento científico innovador y tecnología de vanguardia. También las economías fuertes de los países del primer mundo, han logrado mantener el dominio y control de los sofisticados y complejos procesos de producción y aplicación de la ciencia y la tecnología y, como derivación de ello, amplias posibilidades para resolver los diversos problemas que aquejan a las sociedades, incluyendo los educativos.

Por otro lado, la transición de los distintos modelos de desarrollo social y económico, que pasaron de las burocracias políticas a la inserción de economías neoliberales, recrudeció los antagonismos sociales

\footnotetext{
${ }^{2}$ Los modelos educativos imperantes en estos países, en su generalidad tienden al desarrollo de la persona como individuo y no como integrante de una colectividad en la que comparte intereses y aspiraciones comunes que se traducen en proyectos de vida.
} 
e incrementó la miseria y pobreza de millones de hogares. ${ }^{3}$ Tanto en Europa como en América Latina, se experimentaron procesos democratizadores en la década de los ochenta del siglo pasado, generándose a su vez, alianzas y convenios comerciales entre los Estados-nación para hacer frente a la inminente apertura de las fronteras comerciales, al libre tráfico de los productos con sus repercusiones económicas y a la movilidad constante de los capitales financieros.

En este contexto, la orientación de modelos productivos hacia sistemas cada vez más industrializados y la incorporación de instrumentos avanzados de procesamiento y transferencia de la información, están trasformando sustancialmente los patrones de comportamiento socioculturales de los diversos grupos sociales. Estos cambios que en gran medida son generados por el avance de la ciencia, la innovación tecnológica y los grandes acontecimientos sociales de fin de siglo, están transformando a las sociedades que se encuentran en desarrollo y crecimiento económico y social. Así, las clonaciones, los transgénicos, el proyecto genoma humano, la microcirugía celular, la ingeniería genética, la cibernética y la robótica, son algunos de los resultados de arduos años de trabajo de los grupos de investigadores y tecnólogos que, al aportar sus productos a la sociedad, contribuyen a modificar sustancialmente las distintas esferas de la cotidianidad colectiva, de tal suerte que los tradicionalismos habituales se están refuncionalizando para responder al mundo contemporáneo.

De la misma manera, los últimos acontecimientos en las sociedades modernas y la presencia de nuevos movimientos sociales en los escenarios internacional, regional y local, están modificando los habituales paradigmas de análisis, interpretación y explicación de los procesos socioculturales y como consecuencia de ello, de los sistemas educativos regionales. La desaparición del llamado bloque socialista, el surgimiento de conflictos interétnicos y la agudización de los fundamentalismos religiosos en la Europa del Este, la instauración de regímenes democráticos en los países que tenían gobiernos autoritarios y el ascenso de partidos políticos de tendencia derechista en los nuevos gobiernos, el agotamiento de la vía armada como opción para solucionar los problemas sociales en América Latina y el movimiento zapatista en el sur de Chiapas, son algunos de los elementos presentes en los escenarios del siglo XXI. Tanto las acciones y comportamientos colectivos, como las formas en que las sociedades asumen y construyen nuevos y diversos valores morales, incluyendo los educativos, demandan repensar las condiciones actuales de la educación. Asociados a lo anterior, han surgido novedosos argumentos teóricos para abordar los complejos problemas de la educación, de sus impactos en la formación de las generaciones venideras, así como de las intencionalidades de constituir a la educación como uno de los baluartes para la dignificación y convivencia de la especie humana.

A su vez, la presencia de estos nuevos escenarios que impactan desde lo micro hasta lo macrosocial, está requiriendo que los sistemas educativos nacionales se adecuen a las finalidades de los modelos educativos dominantes. La aplicación de políticas educativas en los últimos veinte años del siglo pasado, responde a los procesos de globalización y mundialización tanto de las economías como del conocimiento y la información (Chomsky, 1997)

En el caso específico de México, la última reforma educativa implementada en 1992, se inscribe en el escenario de la consolidación del modelo neoliberal y de la necesidad de formar profesionales

\footnotetext{
${ }^{3}$ En el informe presentado en 1999 por el Programa de las Naciones Unidas para el Desarrollo Humano (PNUD), se indica que casi 300 millones de personas viven con menos de un dólar diario y aproximadamente mil millones no satisfacen sus necesidades básicas.
} 
capacitados que dominen nuevas habilidades y destrezas intelectuales y prácticas (Rincón, 2000) para responder a los procesos productivos. La educación que se ha constituido en una preocupación de primer orden para que la humanidad progrese en el siglo XXI hacia ideales de paz, libertad y justicia en las naciones (Delors, 1996), también se ha convertido en uno de los grandes problemas que deben resolverse en las sociedades contemporáneas. Es por ello que, la intervención de los investigadores en todos y cada uno de los diversos problemas educativos ${ }^{4}$ que se acentúan continuamente en las naciones pobres, incluido México, requiere partir de la reflexión disciplinaria de las ciencias de la educación y de la participación racional de la investigación científica en el campo educativo con el propósito de formar investigadores que encuentren las vías de solución. Ante este panorama, la formación de profesionales de la educación para la investigación, generación y aplicación de conocimiento innovador, se constituye en una prioridad de la problemática educativa para la consolidación del campo disciplinario de las ciencias de la educación y de la intervención de los complejos problemas que le afectan a la mayoría de los pueblos de América Latina.

\section{¿HACIA DÓNDE ORIENTAR LA FORMACIÓN DE INVESTIGADORES EDUCATIVOS?}

Si la constitución de las ciencias de la educación como disciplinas autónomas e independientes, representa una dificultad para el conocimiento disciplinario, la formación de investigadores para la misma también está matizada por los mismos conflictos y contradicciones que se han expresado en el desarrollo de estos saberes. ¿Cómo formar investigadores en un campo específico como el educativo, donde la definición del objeto de estudio se encuentra caracterizada por indefiniciones y confusiones epistemológicas? ¿Qué tipo de investigadores se deben formar para responder a los nuevos contextos internacionales donde los aportes de la ciencia y la tecnología están teniendo cambios y transformaciones cada vez más agresivos, obligando a lo educativo a acelerar los procesos de formación, de generación y aplicación del conocimiento? ¿Qué papel deben desempeñar los nuevos investigadores educativos ante los desafíos contemporáneos del nuevo milenio? ¿Cómo deben responder ante la existencia de modelos educativos neoliberales que tienden a enfatizar la formación individual en detrimento de la formación colectiva? ¿Qué tipo de problemas deben considerar como prioritarios los investigadores educativos: los que les demanden las políticas educativas gubernamentales o los que están afectando a los sectores sociales más desprotegidos?

Estas interrogantes inducen a reflexionar si la formación de los investigadores educativos, tiene que planificarse a partir de una posición exterior a la educación, o en y dentro de la educación. Mientras por un lado formar investigadores con una visión externa de los conflictos de la educación, significa continuar reproduciendo el viejo esquema de la acción investigativa, donde desde afuera de las ciencias de la educación, diversas disciplinas científicas tanto de las ciencias sociales como de otras ciencias, incluso exactas, -que históricamente no tenían relación con la educación- identifican como objetos de estudio a los problemas de la educación y los abordan con las perspectivas metodológicas y los fundamentos teóricos de sus propias disciplinas. Esta posición plantea reflexionar lo educativo ajeno a los campos disciplinarios que han tenido desarrollos diversos, con rutas teórico-metodológicas que constituidas en la confrontación paradigmática de otras disciplinas, y que asumen a la educación como un agregado más de sus

Programa de las Naciones Unidas para el Desarrollo, Informe sobre desarrollo humano 1999, New York, Ediciones Mundi-Prensa, 1999.

4 Analfabetismo, deserción y abandono escolar, baja eficiencia terminal en los diferentes tipos educativos, bajos índices de aprovechamiento escolar, desarticulación entre cada uno de los tipos educativos, imposición de modelos homogéneos en la mayoría de los país, desvinculación entre la formación profesional y los sistemas productivos, por citar algunos de ellos. 
investigaciones y no como parte esencial de la consolidación de los saberes científicos de las ciencias de la educación. Esta perspectiva de la investigación educativa, significa generar procesos de formación de investigadores ubicados en escenarios externos a los problemas de la educación. Es decir, estudian los problemas desde un escenario distante a lo educativo y justifican y legitiman la implantación de los modelos educativos vigentes.

Por otra parte, discutir la formación de investigadores en y dentro de la educación, tiene una connotación diferente que se asocia al proceso de desarrollo de las ciencias de la educación y a la definición de la educación como su objeto de estudio. Desde este planteamiento, la participación teórica de las diversas disciplinas debe ser entendida como una acción interdisciplinaria y la intervención metodológica y técnica como parte esencial de la colaboración multidisciplinaria. Por tanto, inter y multidisciplinariedad se deben constituir en el eje formativo de los investigadores educativos.

Desde este enfoque, la investigación educativa adquiere un significado distinto que permite un abordaje disciplinario integral y globalizador, de tal suerte que los diversos problemas de la educación se estudien, analicen y reflexionen como un conjunto estructurado que está mediado por múltiples factores que oscilan entre lo económico, político, social, ideológico y cultural, tanto individual como socialmente. Estamos hablando entonces de formar un investigador educativo integral y polivalente, con amplios dominios no sólo en los campos de la pedagogía y la didáctica, sino también de la sociología, economía, política, filosofía, psicología y de cualquier otra ciencia que pueda contribuir a la solución de los problemas de la educación. Sin embargo, cuatro principios formativos deben estar presentes en todo el proceso de formación de investigadores educativos: principios pedagógicos, principios filosóficos, principios sociológicos y principios psicológicos. Cada uno de ellos, contribuirá a formar investigadores capaces de identificar problemas concretos, abordarlos críticamente para presentar propuestas de solución a los más graves problemas educativos de la sociedad; además, orientar y dirigir proyectos de investigación educativa y, esencialmente, procesos de formación de nuevos investigadores.

Este enfoque de la formación de investigadores educativos, también está vinculado a las formas de enseñar a hacer investigación (Sánchez Puentes; 1995) Para orientar estos procesos, no debe evadirse la reflexión de las diversas acciones didácticas que se emplean en la enseñanza de la investigación educativa. En el modelo convencional para formar investigadores, sustentado en los estudios de posgrados, la intervención inter y multidisciplinaria deben favorecer la participación de la didáctica como campo disciplinario coadyuvante de la formación. Asimismo, los cursos teóricos, seminarios metodológicos y talleres técnicos, forman parte de una estrategia integral para la adquisición de habilidades y capacidades tanto teóricas como metodológicas y técnicas. Cada una de estas unidades de enseñanza y aprendizaje se articulan entre sí, a partir del objeto de estudio y teniendo como eje central de la misma formación los problemas de investigación educativa. Los contenidos teóricos disciplinarios se integran al debate metodológico del proyecto de investigación, como parte articuladora de todo el proceso de formación. Así, en cada una de las unidades didácticas del programa de posgrado, el problema de investigación y su objeto de estudio, son abordados tomando como referencia diferentes enfoques teórico-conceptuales que permitan mayor comprensión del estado problemático del objeto de estudio.

Por su parte, el personal docente de los programas de posgrado se integrará en cuerpos colegiados, abandonando el trabajo individual y descontextualizado. La participación de ellos durante el proceso, será una respuesta a las necesidades y requerimientos de dirección y coordinación de los trabajos 
de investigación de los estudiantes. Se incorporará a un proceso integral de desarrollo de la personalidad, en el que las herramientas intelectuales para hacer investigación educativa son algunos de los elementos necesarios y complementarios para formar un profesional de calidad; no un requisito administrativo para justificar las disposiciones institucionales que pretenden justificar el otorgamiento de grados académicos. Esta propuesta significa que cada uno de los docentes, no sólo debe impartir los contenidos propios de las unidades didácticas de enseñanza, además, deberá asumir la función de asesor científico, capaz de dirigir y orientar la formación, tanto de la disciplina específica como del campo de la metodología de la investigación. Así, la figura de asesor científico cobra sentido durante el proceso, así como en cada una de las etapas del mismo, ya que su participación pretende garantizar una formación teórico-conceptual en los estudiantes y la culminación del trabajo de investigación. Su accionar didáctico no sólo se limitará a impartir el curso del cual es el responsable directo, sino, además, debe apoyar y asesorar a los estudiantes para resolver las dificultades que se presenten en el dominio de las diversas disciplinas; pero esencialmente para el manejo de las herramientas instrumentales y las habilidades metodológicas necesarias para el quehacer investigativo. El trabajo colegiado implica llevar un seguimiento de cada estudiante, además de facilitar a los asesores científicos su participación en la formación integral de los profesionistas.

En esta perspectiva formativa, los contenidos teóricos disciplinarios de cada unidad de aprendizaje se integran con el accionar cotidiano de los asesores científicos y el desarrollo de la metodología de la investigación, se consolida con la interacción entre los conocimientos conceptuales y los conocimientos prácticos, empíricos y metodológicos. Una formación para la investigación en educación, vista desde la participación colegiada y la integración de los contenidos educativos, puede constituirse en una respuesta a las limitaciones que actualmente se han presentado en los modelos tradicionales de formación de investigadores educativos.

\section{CONCLUSIONES}

La formación de investigadores en educación en América Latina, se encuentra precedida por el desarrollo de los campos disciplinarios que abordan a la educación como su objeto de estudio y por la presencia de modelos educativos neoliberales. Por lo tanto, también el estado actual de la investigación educativa en nuestros países, se encuentra influido por las formas en que las diferentes disciplinas científicas, incluidas las llamadas ciencias de la educación, han logrado un nivel de consolidación teórico y metodológico en la búsqueda de encontrar soluciones a los problemas educativos. Además, la producción del conocimiento educativo, tiene que enfrentar los desafíos de los grandes cambios en la ciencia, la técnica y la sociedad. Cambios que exigen reorientar los procesos de formación de investigadores.

Es precisamente en este panorama que los modelos tradicionales de formación (programas escolarizados de posgrado) tienen que competir con otras opciones, tales como la educación virtual, a distancia o los programas institucionales como PROMEP5. Sin embargo, independiente de que si el modelo de formación es escolarizado, virtual, a distancia o como lo plantea el PROMEP, una posibilidad para formar un profesional calificado para la investigación educativa, es la integración inter y multidisciplinaria de los diferentes campos científicos que han abordado a lo educativo como objeto de estudio. A la par de esta

\footnotetext{
${ }^{5}$ El Programa de Mejoramiento del Profesorado que se ha instituido en las Instituciones de Educación superior públicas en México, es uno de los tantos programas que se están aplicando para mitigar los bajos salarios de los docentes universitarios. En el caso particular de la educación básica, en este país, se ha instrumentado el programa de Carrera Magisterial que también tiene como propósito amortiguar los efectos de la crisis económica, vía incentivos económicos.
} 
integración, la colaboración colegiada de equipos de académicos se hace imprescindible para contribuir a la formación de un investigador con amplias posibilidades y capacidades metodológicas, teóricas, pedagógicas, psicológicas, filosóficas y sociológicas. Es decir, formar a los investigadores de la educación desde el interior de los problemas mismos y no desde posiciones que se encuentren fuera de los mismos.

\section{BIBLIOGRAFÍA}

ANUIES, Catálogo de posgrado 1999, México, Dirección de Servicios Editoriales, 1999.

Confluencia, Órgano informativo de la Asociación Nacional de Universidades e Instituciones de Educación Superior, Año 8, junio de 2000, № 88, México.

EI PROMEP etapa de planeación: enero de 1997-abril de 1999, México, ANUIES, Dirección de Servicios Editoriales.

BRUNNER, José Joaquín, "La investigación educativa en América Latina: presente y futuro" en Universidad Futura, México, UAM-Azcapotzalco, Vol. 3, núms. 8 y 9, pp.92-105.

CONACyT, 1999, Indicadores de actividades científicas y tecnológicas, 1998, México, México, CONACyT. 2000, Padrón de Programas de Excelencia, México, CONACyT.

Página www.conacyt.mx/sni/sni0024.html

DELORS, Jaques, 1996, La educación encierra un tesoro, Madrid, UNESCO, Santillana.

DE Landsheere, Gilber, 1996, La investigación educativa en el mundo, México, Fondo de Cultura Económica,.

GIL Antón, Manuel et. al 1994, Los rasgos de la diversidad, México, UAM-Azcapotzalco.

KUHN, Thomas S. 1975, La estructura de las revoluciones científicas, Madrid, I.C.E.

NOAM, Chomsky y Heinz Dieterich, 1997, La sociedad global, México, Editorial Joaquín Mortiz.

MARTÍNEZ Rizo, Felipe, 1997, El oficio del investigador educativo, Aguascalientes, Universidad Autónoma de Aguascalientes, segunda edición.

ORGANIZACIÓN de las Naciones Unidas, 1999, Programa de las Naciones Unidas para el desarrollo, New York, Ediciones Mundi-Prensa.

PACHECO Méndez, Teresa y Ángel Díaz Barriga, 1997, La profesión. Su condición social e institucional, México, CESU-UNAM-Porrúa Editores.

RINCÓN Ramírez, Carlos, 2000, Construcción del objeto de estudio. Punto de partida del proyecto de investigación, Tuxtla Gutiérrez, UPN-Unidad Tuxtla Gutiérrez.

2000, Naturaleza política de la modernización de la educación básica: realidades y desafíos, Tuxtla Gutiérrez, UNACH.

SÁNCHEZ Puente, Ricardo, 1995, Enseñar a investigar. Una didáctica nueva de la investigación científica en ciencias sociales y humanas, México, CESU-UNAM, ANUIES.

SEP, 1999, PROMEP Apoyo a profesores con perfil deseable. Convocatoria 1999, México, SESIC. 
SOUZA Silva, José de, El cambio de época, el modo emergente de producción de conocimiento y los papeles cambiantes de la investigación y la extensión en la academia del siglo XXI, ponencia presentada en la 1르 Conferencia Interamericana de Educación Agrícola Superior y Rural, organizado por el Instituto Interamericano de Cooperación Agrícola, Panamá, 16-19 de noviembre de 1999.

UNICEF, Estado mundial de la infancia 1999. Educación, material página en Internet: www.unicef.org/spanish.

VILLASEÑOR, Guillermo (coord.), 1997, La identidad de la educación superior en México, México, Coordinación de Humanidades, Centro de Estudios Sobre la Universidad, Universidad Autónoma Metropolitana-Xochimilco, Universidad Autónoma de Querétaro. 